\title{
Locality of the overlap-Dirac operator on topology-fixed gauge configurations
}

\section{Yong-Gwi Cho*}

Graduate School of Pure and Applied Sciences, University of Tsukuba

Tsukuba, Ibaraki 305-8571, Japan

E-mail: Chodccs.tsukuba.ac.ip

\section{Shoji Hashimoto}

High Energy Accelerator Research Organization (KEK) and

School of High Energy Accelerator Science, The Graduate University for Advanced Studies

Tsukuba 305-0801.

E-mail: shoji.hashimotodkek.jp

\begin{abstract}
We investigate the locality property of the overlap-Dirac operator on gauge configurations generated with extra Wilson fermions. By such extra terms we expect that the structure of the Aoki phase would change drastically. In particular, we study the possibility of defining the overlapDirac operator in the strong coupling regime keeping its exponential locality.
\end{abstract}

The 30th International Symposium on Lattice Field Theory

June 24-29, 2012

Cairns, Australia

\footnotetext{
*Speaker.
} 


\section{Introduction}

Locality of the overlap-Dirac operator is not obvious, since its definition [U]

$$
a D_{o v}=1+\gamma_{5} \frac{H_{W}}{\left|H_{W}\right|},
$$

includes an operator $H_{W}$ in the denominator. Here, $H_{W}$ is the hermitian Wilson-Dirac operator $H_{W}=\gamma_{5} D_{W}$, that is used as a kernel to construct $D_{o v}$. Superficially, if the eigenvalue spectrum of $H_{w}$ contains near-zero modes, the overlap-Dirac operator may violate locality.

The locality is known to be satisfied at weak couplings. To be specific, on background gauge configurations satisfying some smoothness condition, one can show that $D_{o v}$ is exponentially localized []], i.e. $\left|\left(D_{o v}\right)_{x y}\right| \leq \exp (-|x-y| / \ell)$ with a localization length $\ell$. This condition is however too strong for practical setup used in present lattice QCD simulations; numerical tests are necessary for more realistic cases.

Golterman and Shamir conjectured that the overlap-Dirac operator defined outside of the Aoki phase is local [B]]. Contrary to the original argument [田], the Aoki phase in this case defined by the profile of near-zero modes: inside the Aoki phase the near-zero modes are extended in space, while they are localized outside. The value of eigenvalue $\lambda_{c}$ above which the eigenmodes are extended is called the mobility edge borrowing the terminology of condensed matter physics. Then, the localization length of the overlap-Dirac operator is determined either by $1 / \lambda_{c}$ or $\ell(\lambda)\left(|\lambda|<\lambda_{c}\right)$, where $\ell(\lambda)$ is the localization length of the individual low-lying modes. Thus, the question of the locality crucially depends on the background gauge field.

The origin of the near-zero modes of $\left|H_{W}\right|$ is the roughness of the gauge configuration. A simple analytic example is given in [可]. Therefore, the localization length is expected to increase toward strong couplings or coarse lattices, and the definition of the overlap-Dirac operator becomes more difficult. To avoid this problem one may introduce additional terms to the lattice action, such as those proposed in [目]. They consist of two flavors of heavy Wilson fermions and their associated ghosts carrying a twisted mass term

$$
S_{e x}=\sum_{x} \bar{\chi}(x) D_{W}\left(m_{0}\right) \chi(x)+\sum_{x} \bar{\phi}(x)\left[D_{W}\left(m_{0}\right)+i \mu \gamma_{5} \tau_{3}\right] \phi(x),
$$

where $\chi$ denotes Wilson fermions with a negative mass $m_{0}$. The second term represents bosonic fields to cancel the bulk of the effects of Wilson fermions. In fact, the action generates a suppression factor

$$
\operatorname{det}\left[\frac{H_{W}\left(m_{0}\right)^{2}}{H_{W}\left(m_{0}\right)^{2}+\mu^{2}}\right]
$$

in the partition function. Then, the gauge configuration with small eigenvalues of $H_{W}\left(m_{0}\right)$ lower than $\mu$ is suppressed, and the potentially dangerous near-zero modes disappear from the eigenvalue spectrum. Since the low-lying eigenvalue can never cross zero due to the suppression factor $\operatorname{det} H_{W}\left(m_{0}\right)^{2}$, global topology of the gauge field configuration does not change under continuous deformations. The simulations are thus confined in a given topological sector with this action.

In this work, we investigate the spectrum of $H_{W}$ and the locality of $D_{o v}$ in the strong coupling regime with or without the extra Wilson fermion terms. The purpose of the study is to explore the possibility to use the overlap fermion at coarser lattices than currently available ones. 


\begin{tabular}{|c|ccccc|cccc|}
\hline$\mu$ & \multicolumn{5}{|c|}{0} & \multicolumn{4}{c|}{0.2} \\
\hline$\beta$ & 5.95 & 5.83 & 5.63 & 5.50 & 5.43 & 5.78 & 5.68 & 5.48 & 5.28 \\
\hline$a(\mathrm{fm})$ & 0.10 & 0.12 & 0.15 & 0.27 & - & 0.10 & 0.12 & 0.14 & 0.27 \\
\hline
\end{tabular}

Table 1: Lattices parameters

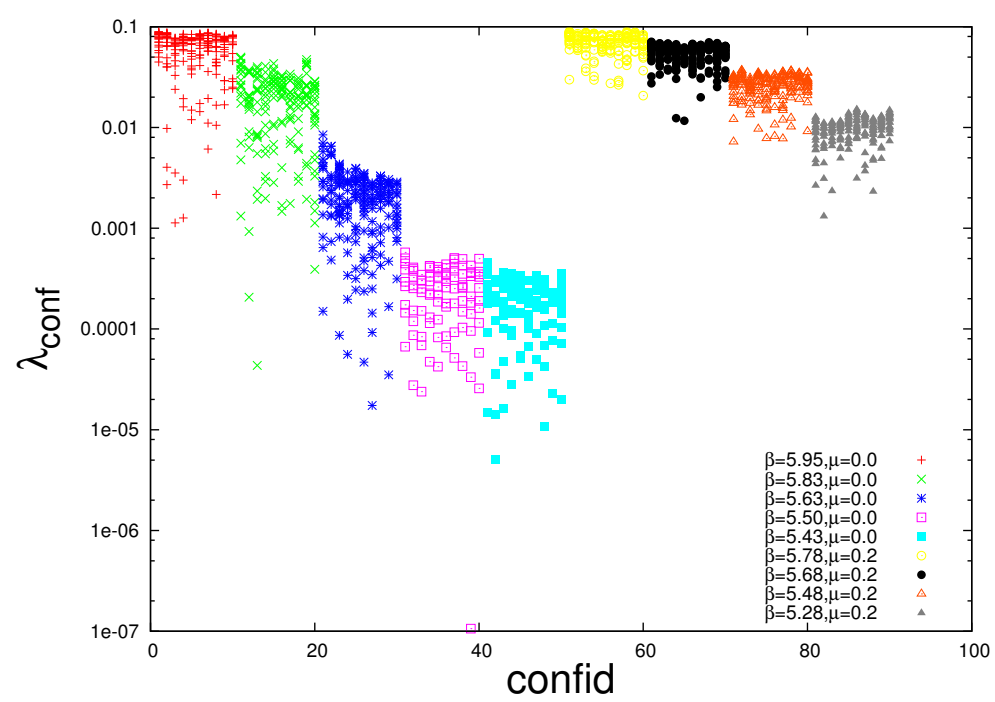

Figure 1: Near-zero eigenvalue distribution of $\left|H_{W}\right|$ plotted in the logarithmic scale. The five clusters on the left show the results for the standard gauge action while the right four clusters are those with the low-mode suppression term.

\section{Eigenvalue distribution}

In this study, we use quenched lattices (no dynamical light fermions) at the $\beta$ values listed in Table $\square$. Lattice size is $16^{3} \times 32$ and the gauge action is the standard Wilson gauge action. We consider the lattices with and without the low-mode suppression term (ㅍ.3). In the Table, the lattices without that term are denoted by $\mu=0$ as the extra factor cancels in this case. The large negative mass $a m_{0}$ is -1.6 , and the number of gauge configurations studied is 10 for each parameter. Lattice spacing $a$ is estimated using the Sommer scale $r_{0}$ extracted from the static quark potential.

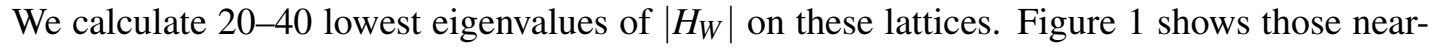
zero eigenvalue distribution in a logarithmic scale. It is clear that the number of low-modes increases on coarser (or smaller $\beta$ ) lattices. With the low-mode suppression term (four right clusters), they are indeed highly suppressed. Compared at similar lattice spacings, the lowest eigenvalue is 1-2 orders of magnitude higher.

According to the Banks-Casher relation [ [ [] , the absence of the low-lying modes implies that the flavor-parity symmetry broken phase as defined by Aoki is not entered for these lattices. This is the effect of the low-mode suppressing term, though the gap is rather small at coarser lattices. The practical question is then how large the localization length is for these coarse lattices, which is addressed in the next section. 

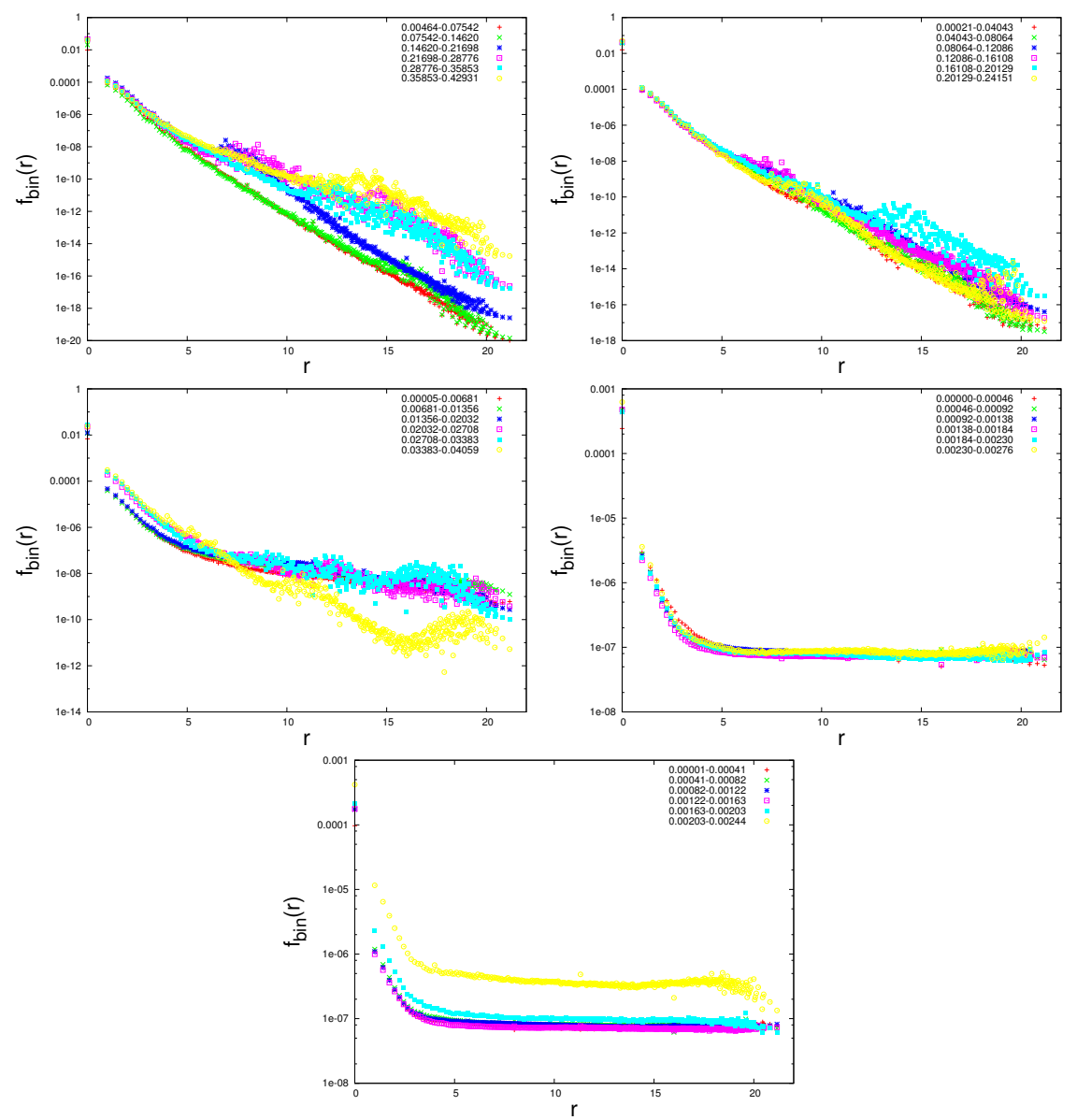

Figure 2: $f_{\text {bin }}(r)$ on the quenched gauge configurations without the low-mode suppressing term $(\mu=0.0)$. The data are shown for $\beta=5.95$ (top left), $\beta=5.83$ (top right), $\beta=5.63$ (middle left), $\beta=5.50$ (middle right), and $\beta=5.43$ (bottom). The eigenmodes are binned in different ranges of the eigenvalues as indicated in the legend of each plot.

\section{Localization of eigenmodes}

In order to investigate the locality of $D_{o v}$ we look at the spatial profile of low-lying modes. For each eigenmodes $\phi_{i}(x)$ of $H_{W}$, we define $\rho_{i}(x)$ and $f_{i}(r)$ as

$$
\begin{aligned}
\rho_{i} & =\phi_{i}^{\dagger}(x) \phi_{i}(x), \rho_{i}\left(x_{0}\right)=\max _{x}\left\{\rho_{i}(x)\right\}, \\
f_{i}(r) & =\left\{\rho_{i}(r)|r=| x-x_{0} \mid\right\}
\end{aligned}
$$

following to [Q] . Namely $\rho_{i}(x)$ is the strength of the mode, and $f_{i}(r)$ represents the profile of that mode as a function of the distance from the position where $\rho_{i}(x)$ has a maximum. In calculating $f_{i}(r)$, different orientations giving the same $r$ are averaged.

In Figure 凤, we plot $f_{i}(r)$ averaged over configurations after binning the eigenmodes in different ranges of their eigenvalues, that we call $f_{b i n}(r)$. At the $\beta$ values above 5.63, we find a clear fall-off of the eigenmodes as a function of $r$. That is true even at the highest bin we measured. This 

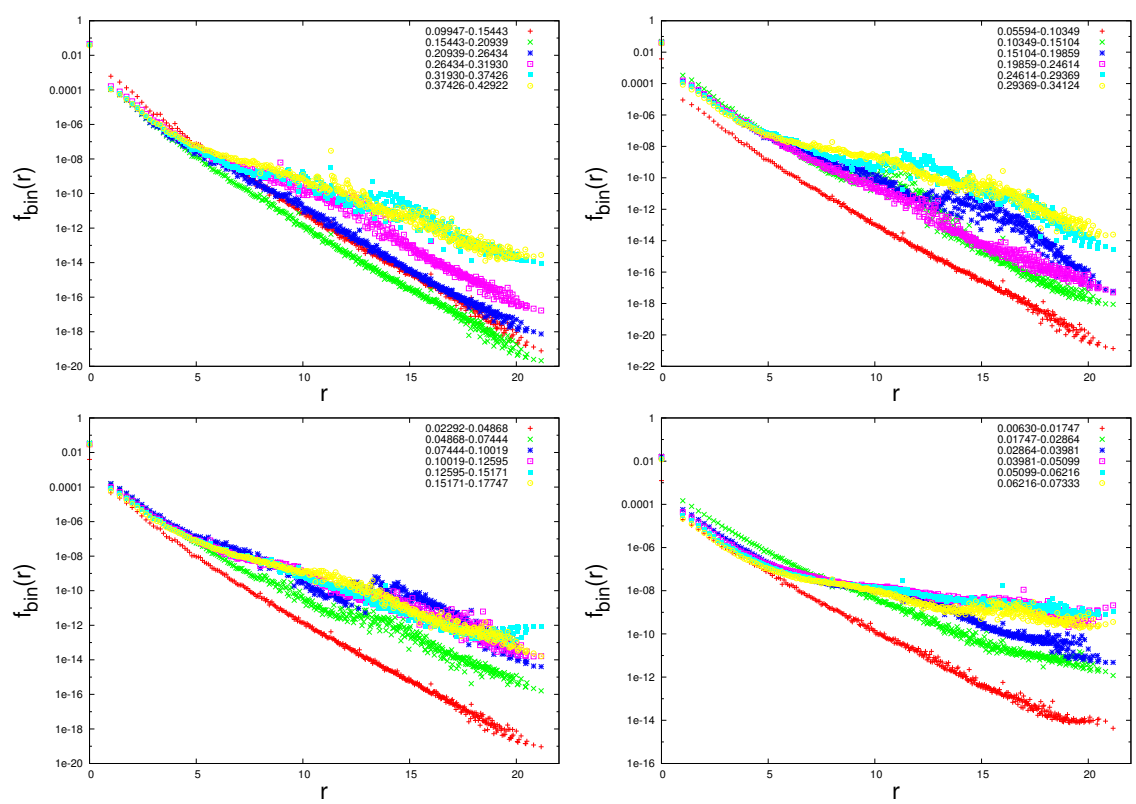

Figure 3: Same as Figure $\square$ but with the low-mode suppression term $(\mu=0.2)$. The data are shown for $\beta=5.78$ (top left), $\beta=5.68$ (top right), $\beta=5.48$ (bottom left), and $\beta=5.28$ (bottom right).

indicates that $D_{o v}$ constructed on these gauge configurations is local with the length controlled by the fall-off of these low-lying modes.

At $\beta=5.50$ and 5.43, on the other hand, we find that $f_{\text {bin }}(r)$ becomes flat beyond $r \simeq 5$. It means that the system is already in the Aoki phase. This results is consistent with results of [एँ]], where the mobility edge falls down to zero at $\beta=5.5$ and $a m_{0}=-1.5$.

Once we introduce the low-mode suppression term, not only the eigenvalue spectrum but the profile of the eigenmode changes as shown in Figure [3. Even at the $\beta$ value as low as 5.28, where the lattice spacing is roughly $0.27 \mathrm{fm}$, the low-lying modes are still localized. This implies that these parameter regions are outside of the Aoki phase. In other words, the Aoki phase structure is drastically changed by the effect of the low-mode suppressing term. The phase structure of the Wilson fermion would thus be changed as illustrated in Figure $\mathbb{\theta}$.

\section{Locality of overlap-Dirac operator}

The localization length of the overlap-Dirac operator $D_{o v}$ can also be extracted directly from the behavior of $D_{o v}$. We first set a source field as

$$
\eta_{\alpha}(x)= \begin{cases}1 & x=(0,0,0,0) \\ 0 & \text { otherwise }\end{cases}
$$

where $\alpha$ is an index of internal degree of freedom for fermions. Then we calculate a norm of a vector $\psi(x)=\operatorname{sgn}\left(H_{W}\right) \eta(x)$

$$
f(r)=\|\psi(x)\|, r=\|x\| .
$$




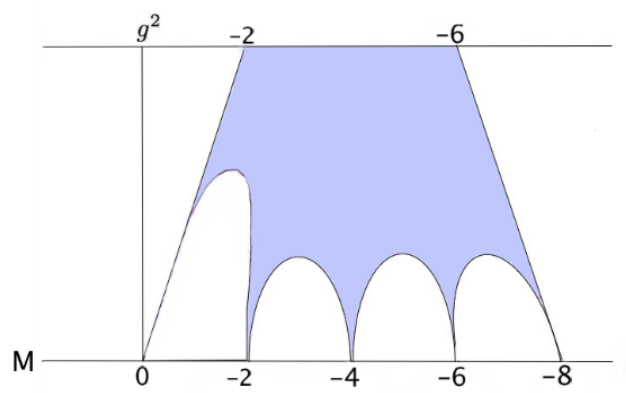

Figure 4: Expected phase structure of the Wilson fermion after the low-mode suppression term is included.

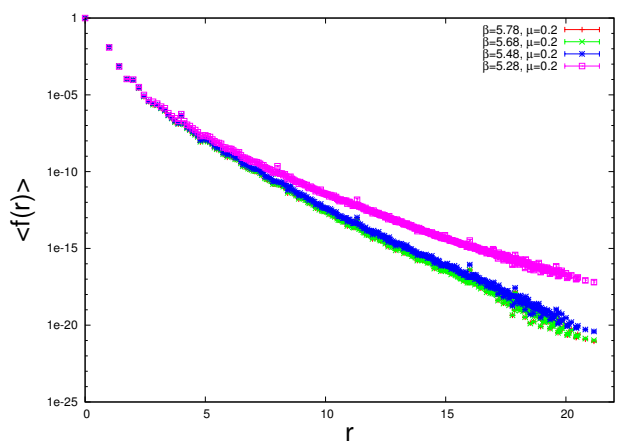

Figure 5: $\langle f(r)\rangle$ as a function of $r$. Data for the low-mode suppressed gauge configurations: $\mu=0.2$.

In Figure [1, we plot $\langle f(r)\rangle$ as a function of $r$ on the gauge configurations generated with the low-mode suppressing term. For all four $\beta$ values calculated, $\langle f(r)\rangle$ is rapidly decaying as distance $r$ increases.

To determine the localization length, we fit $\langle f(r)\rangle$ to an exponential function

$$
\langle f(r)\rangle=c \exp (-r / l),
$$

at large distances $r$. The results for the localization length $l$ is listed in Table $\square$. It shows that the overlap-Dirac operator can be properly defined at strong couplings as far as the low-mode suppressing term is introduced.

\begin{tabular}{|c|c|}
\hline$\beta$ & $l$ (localization length) \\
\hline \hline 5.78 & 0.58 \\
\hline 5.68 & 0.57 \\
\hline 5.48 & 0.60 \\
\hline 5.28 & 0.76 \\
\hline
\end{tabular}

Table 2: Localization length calculated on the gauge configurations with the low-mode suppressing term $\mu=0.2$. 


\section{Conclusion}

We studied the effect of the low-mode suppressing term on the locality of the overlap-Dirac operator. By inspecting the low-lying eigenmodes of $H_{W}$, we find the the mobility edge is finite even at $\beta=5.28$, which corresponds to $a \simeq 0.27 \mathrm{fm}$.

Numerical calculations are performed on Hitachi SR16000 at High Energy Accelerator Research Organization (KEK) under a support of its Large Scale Simulation Program (No. 11-05). SH is supported in part by the Grant-in-Aid of the Japanese Ministry of Education (No. 21674002).

\section{References}

[1] Herbert Neuberger. More about exactly massless quarks on the lattice. Phys.Lett., B427:353-355, 1998.

[2] Pilar Hernandez, Karl Jansen, and Martin Luscher. Locality properties of Neuberger's lattice Dirac operator. Nucl.Phys., B552:363-378, 1999.

[3] Maarten Golterman and Yigal Shamir. Localization in lattice QCD. Phys.Rev., D68:074501, 2003.

[4] Sinya Aoki. New Phase Structure for Lattice QCD with Wilson Fermions. Phys.Rev., D30:2653, 1984.

[5] Federico Berruto, Rajamani Narayanan, and Herbert Neuberger. Exact local fermionic zero modes. Phys.Lett., B489:243-250, 2000.

[6] Hidenori Fukaya et al. Lattice gauge action suppressing near-zero modes of $\mathrm{H}(\mathrm{W})$. Phys.Rev., D74:094505, 2006.

[7] M.F. Atiyah and I.M. Singer. Dirac Operators Coupled to Vector Potentials. Proc.Nat.Acad.Sci., 81:2597-2600, 1984.

[8] Tom Banks and A. Casher. Chiral Symmetry Breaking in Confining Theories. Nucl.Phys., B169:103, 1980. Revised Version.

[9] N. Yamada et al. Mobility edge and locality of the overlap-Dirac operator with and without dynamical overlap fermions. PoS, LAT2006:060, 2006.

[10] Maarten Golterman, Yigal Shamir, and Benjamin Svetitsky. Localization properties of lattice fermions with plaquette and improved gauge actions. Phys.Rev., D72:034501, 2005. 